\title{
Effects of the $\mathrm{N}$ terminus of mouse DNA polymerase $\kappa$ on the bypass of a guanine-benzo[a]pyrenyl adduct
}

\author{
Received July 13, 2015; accepted November 14, 2015; published online December 3, 2015
}

\section{Yang Liu*, Xiaolu Ma and Caixia Guo ${ }^{\dagger}$}

Key Laboratory of Genomic and Precision Medicine, China Gastrointestinal Cancer Research Center, Beijing Institute of Genomics, Chinese Academy of Sciences, Beijing 100101, China

*Yang Liu, Key Laboratory of Genomic and Precision Medicine, China Gastrointestinal Cancer Research Center, Beijing Institute of Genomics, Chinese Academy of Sciences, No.1 Beichen West Road, Chaoyang District, Beijing 100101, China. Tel: 010-86-84097678, email: liuyang@big.ac.cn

${ }^{\dagger}$ Caixia Guo, Key Laboratory of Genomic and Precision Medicine, China Gastrointestinal Cancer Research Center, Beijing Institute of Genomics, Chinese Academy of Sciences, No.1 Beichen West Road, Chaoyang District, Beijing 100101, China. Tel: 010-86-84097646, email: guocx@big.ac.cn

DNA polymerase $\kappa$ (Polк), one of the typical member of the Y-family DNA polymerases, has been demonstrated to bypass the $10 S(+)$-trans-anti-benzo[a]pyrene diol epoxide- $\boldsymbol{N}^{2}$-deoxyguanine adducts (BPDE-dG) efficiently and accurately. A large structural gap between the core and little finger as well as an N-clasp domain are essential to its unique translesion capability. However, whether the extreme N-terminus of Polк is required for its activity is unclear. In this work, we constructed two mouse Polk deletions, which have either a catalytic core $\left(\mathrm{mPolk}_{1-516}\right)$ or a core without the first 21-residues $\left(\mathrm{mPol \kappa}_{22-516}\right)$, and tested their activities in the replication of normal and BPDEDNA. These two Polk deletions are nearly as efficient as the full length protein $\left(\right.$ Polk $\left._{1-852}\right)$ in normal DNA synthesis. However, steady-state kinetics reveals a significant reduction in efficiency of dCTP incorporation opposite the lesion by Polk ${ }_{22-516}$, along with increased frequencies for misinsertion compared with $\mathrm{Pol \kappa}_{1-852}$. The next nucleotide insertion opposite the template $\mathrm{C}$ immediately following the BPDE-dG was also examined, and the bypass differences induced by deletions were highlighted in both insertion and extension step. We conclude that the extreme N-terminal part of Polk is required for the processivity and fidelity of Polк during translesion synthesis of BPDE-dG lesions.

Keywords: translesion DNA synthesis/Y-family DNA polymerase $\kappa /$ polycyclic aromatic hydrocarbons/ BPDE-dG lesions/enzyme fidelity.

The human genome is exposed to a variety of endogenous and exogenous stresses that lead to DNA damages. Although most damaged DNAs are efficiently repaired by cellular repair system, those that escape repair strongly affect DNA replication in the $\mathrm{S}$ phase. Translesion DNA synthesis (TLS) is one of the DNA damage tolerance processes. It employs specialized TLS polymerases to synthesize DNA across and beyond a variety of replication-blocking lesions, thus avoiding replication fork collapse (I). Up to date, eight translesion synthesis polymerases have been identified in mammals, and four of them, namely Poln, Polt, Polא and REV1 belong to the Y-family (2-4).

Due to the different nature and structure of DNA adducts, every Y-family member exhibits distinct preference for specific DNA lesions (5). For example, the eukaryotic Pol $\eta$ is specialized in accurately bypassing cyclobutane pyrimidine dimer (CPD) with insertion of two dATP opposite a TT dimer $(6,7)$. Crystal structure studies show that Pol $\eta$ functions as molecular splint to stabilize the TT dimer in a normal B-form conformation, and the CPD lesion is well accommodated in the active site for translesion synthesis (8). Deficiency of Poln leads to a variant form of xeroderma pigmentosum diseases $(9,10)$. Although Polı could not replicate the TT dimer (11), it has been suggested to be involved in the error free (12) or error prone (13) replication of certain $\mathrm{N}^{2}$-deoxyguanine $(\mathrm{dG})$ adducts, such as $1, \mathrm{~N}^{2}$-propano-2'deoxyguanosine $(\gamma-\mathrm{HOPdG})$ resulting from lipid peroxidation or $\mathrm{N}^{2}-\mathrm{dG}$ adducts derived from 2-amino-3-methylimidazo[4,5-f]quinoline, respectively. Recently, based on reported germline variations in human Single Nucleotide Polymorphism database, several genetic variants of Polt have been constructed and their biochemical activities of bypassing different lesions, such as abasic site, 8-oxoG, $\mathrm{O}^{6}$-MeG and $\mathrm{N}^{2}$-EtG lesion, have been examined (14). These data suggest that certain genetic variations in the TLS polymerases affect their nucleotide incorporation in vitro (14). REV1, which is unable to support TLS across TT dimers or [6-4] photoproducts, acts as scaffold protein and plays roles in regulating other TLS polymerases' activities (15). Polk, the only Y family member with homologs in both bacteria (Dinb and Pol IV) and archaea (Dpo4 and Dbh), is most ubiquitous among eukaryotic Y-family polymerases.

A classic polymerase catalytic core consisting of palm, thumb and finger domains and an additional little finger (LF) domain are present in all Y-family polymerases. A N-clasp subdomain, which covers residues 21-74 and augments the conventional righthanded grip on the primer-template by the palm, fingers and thumb domains is observed in Polк (16). The catalytic core of human apo Polк with the first 18-67residue removed (19-526 and 68-526 aa) have been studied by X-ray crystallography either alone (16) or in complex with a normal template-primer and an incoming nucleotide (17). Although residues 19-32 are disordered in the crystal structure, residues 33-74 encircle a normal primer/template pair (17) and residues 19-68 


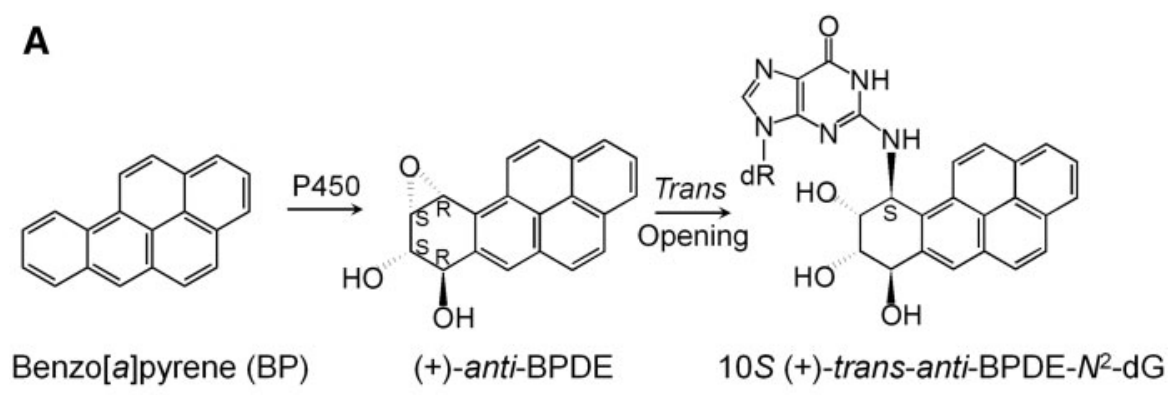

(BPDE-dG)

\title{
B
}

\author{
22/43-nt duplex \\ 5'-GCATAGGGCGAATTCGGATGGT \\ 3'-CGTATCCCGCTTAAGCCTACCATCXCTATATGTCATGCATCAG
}

\author{
24/43-nt duplex \\ 5'-GCATAGGGCGAATTCGGATGGTAG \\ 3'-CGTATCCCGCTTAAGCCTACCATCXCTATATGTCATGCATCAG
}

\author{
25/43-nt duplex \\ 5'-GCATAGGGCGAATTCGGATGGTAGC \\ 3'-CGTATCCCGCTTAAGCCTACCATCXCTATATGTCATGCATCAG
}

\section{$X=d G$ or $B P D E-d G$}

Fig. 1 (A) Structures of benzo[a]pyrene (BP), its metabolite $(+)-(7 R, 8 S, 9 S, 10 R)$ diol epoxide (+)-anti-BPDE) and the $10 S(+)$-trans-antiBPDE- $N^{2}-\mathrm{dG}\left(10 S(+)\right.$-trans-anti-[BP]- $\left.N^{2}-\mathrm{dG}\right)$ adduct. (B). Sequence context employed in the assay. The running-start assay was done by $22 / 43-$ nt duplex, and the standing-start experiment for single nucleotide incorporation opposite the G (or BPDE-dG adduct) and the template base $\mathrm{C}$ immediately following the G (or BPDE-dG adduct) is done by 24/43-nt and 25/43-nt duplexes, respectively.

is required for DNA synthesis from matched and mismatched primer-template termini (17). Human Polк (hPolk) with removal of either the first 67-aa (68-526) or 90-residue (91-559) has attenuated catalytic activity over mismatched primer termini compared with the catalytic core (1-526) or the core minus the first 18 residues (19-526) $(16,17)$. At present, whether residues 1-18 of the extreme N-terminus of hPolk are required for its TLS function remain unclear.

$(+)-7 \mathrm{R}, 8 \mathrm{~S}, 9 \mathrm{~S}, 10 \mathrm{R}$ - benzo[ $a]$ pyrene dihydrodiol epoxide $[(+)$-anti-BPDE] is the most mutagenic and tumorigenic metabolite of benzo $[a]$ pyrene $(\mathrm{B}[a] \mathrm{P})$ (Fig. 1A), the representative member of polycyclic aromatic hydrocarbons (PAHs) that is present in polluted air and in tobacco smoke (18). (+)-anti-BPDE reacts readily with the exocyclic amino groups of guanine residues in DNA forming the major (+)trans-anti-BPDE- $\mathrm{N}^{2}-\mathrm{dG}$ adducts (abbreviated as BPDE-dG) (Fig. 1A) (19). Several in vivo and/or in vitro studies have identified that BPDE-dG adduct might among the cognate or the most preferred lesion substrates for Polк. Polк can perform error-free bypass of the bulky minor groove (+)-BPDE-dG adduct with incorporating correct $\mathrm{dCTP}$ opposite the lesion (20-22). The uniquely large structural gap between the core and LF domain in Polk is demonstrated to be essential for accommodating minor groove $(+)$-BPDE-dG adduct and for efficient bypass (23). mPolк with the first 51 residues deleted (52-516) leads to failure of BPDE bypass but not for normal DNA synthesis (23). In correspondence to in vitro analyses, Polk-deficient mouse embryo fibroblasts and embryo stem cells are sensitive to benzo $[a]$ pyrene treatment and exhibit increased BPDE-induced mutagenesis (24-27).

In this study, we analysed whether the extreme $\mathrm{N}$-terminus of mPolk (1-21), which is equivalent to resi-

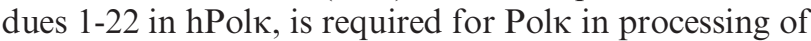
the bulky minor groove BPDE-adduct. To answer that, we constructed Pol $\kappa_{1-516}$ and Pol $\kappa_{22-516}$ (Fig. 2A) and compared their ability to replicate DNA opposite BPDE modified $\mathrm{dG}$ as well as undamaged DNAs. Our results reveal that the extreme $\mathrm{N}$-terminal part of Polk is required for its bypass of BPDE-dG lesions, and both the replication fidelity and the processivity are greatly affected by the modifications.

\section{Experimental Procedures}

\section{Materials}

All chemicals were analytical grade. The enzyme OptiKinase was obtained from USB Molecular Biology Reagents and Biochemicals (Cleveland, OH). The T4 DNA ligase was obtained from New 

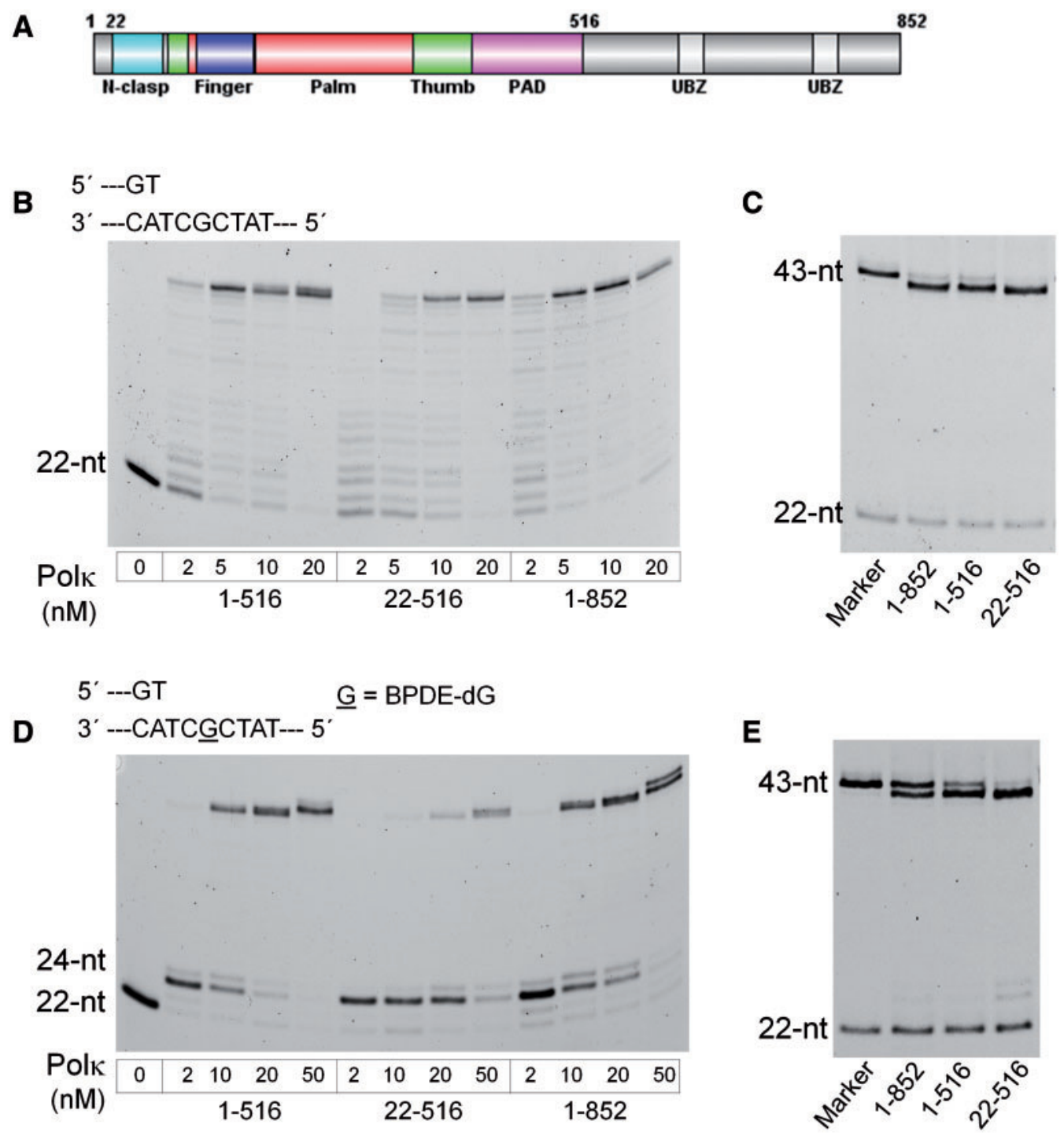

Fig. 2 Primer extension opposite normal and (+)-trans-anti-BPDE- $N^{2}$-dG templates by the full-length and truncated $\mathrm{mPol \kappa}$. (A) The structure domains of mPolk. The schematic diagram is drawn by DOG (v.2.0) (38), with truncation sites indicated. (B) Running-start primer extension experiments on normal DNA template. Reactions were carried out in the presence of all four dNTPs for 5 min at $37^{\circ} \mathrm{C}$ with increased concentrations of Polк as shown below each track. (C) Analysis of extension product lengths over normal dG template by full-length and truncated mPolк. 43-nt marker sequence as well as 22-nt primer is in a separate lane. The sequence context of the 43-nt marker, which corresponds to the primer full extension product strand in Figure 1B, is listed in the 'Materials' section. The reactions were catalysed by $20 \mathrm{nM}$ Polк at $37^{\circ} \mathrm{C}$ for 10 min. (D) Running-start primer extension experiments on BPDE-dG template. Reactions were carried out with increasing concentrations of Polk at $37^{\circ} \mathrm{C}$ for $10 \mathrm{~min}$. The underlined $\mathrm{G}$ represents the BPDE-dG lesion. The 22 -nt primer was readily extended to the two nucleotides $3^{\prime}$ of the lesion and stalled at template position 24-nt, immediately before the lesion. (E) Comparison of primer extension products length catalysed by

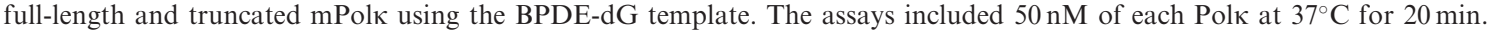

England BioLabs (Ipswich, MA). The dNTPs were also purchased from NEB. The 5' 6-carboxyfluorescein (6-FAM)-labelled 22-nt primer 5'-GCATAGGGCGAATTCGGATGGT-3', 24-nt primer $5^{\prime}$-GCATAGGGCGAATTCGGATGGTAG-3' and 25-nt primer $5^{\prime}$-GCATAGGGCGAATTCGGATGGTAGC-3' (Fig. 1B) were purchased from Life Technologies (Shanghai, China). The $5^{\prime}$ 6-carboxyfluorescein (6-FAM)-labelled 43-nt marker sequence $5^{\prime}-\mathrm{G}$ CATAGGGCGAATTCGGATGGTAGCGATATACAGTACGT AGTC- $3^{\prime}$ was purchased from Generay BioTechnologies (Shanghai, China). The unmodified oligonucleotides were obtained from Sangon, Inc. (Shanghai, China). The oligonucleotides were further purified by $20 \%$ denaturing polyacrylamide gel electrophoresis (PAGE) containing 7.5 M Urea. The (+)-trans-BPDE-dG lesion embedded in 11-nt sequence 5'-ATATCGCTACC-3' (the underlined $\mathrm{G}$ represents the BPDE- dG lesion) is generated as previously described (28). The 11-nt sequence is further ligated to the left 13nt (5'-GACTACGTACTGT-3') and right 19-nt (5'-ATCCGAATTC GCCCTATGC- $3^{\prime}$ ) forming the final 43 -nt products in the context of

\section{(5'-GACTACGTACTGTATATCG GTACCATCCGAATTCGCC CTATGC-3') (Fig. 1B).}

\section{Purification of the full length and truncated mPolk proteins}

Full-length mPolk-pET-16b expression vector was constructed as described previously (29). The catalytic core $\mathrm{mPol}_{1-516}$ and $\mathrm{N}$-terminal truncated $\mathrm{mPol}_{22-516}$ constructs were generated by PCR amplification. Each construct was sequenced correctly. The proteins were expressed and purified as described in (23).

\section{Running-start primer extension assay by the full-length and} truncated $m$ Polk in vitro

For in vitro primer extension experiments, the unlabelled and the $5^{\prime}$ - end labelled 6-FAM-primer 22-nt 5'-GCATAGGGCGAATTC GGATGGT-3' were annealed to the template strand 5'-GACTAC GTACTGTATATCXCTACCATCCGAATTCGCCCTATGC-3' at a molar ratio of $1: 1.2$, where $\mathrm{X}$ is $\mathrm{dG}$ or BPDE-dG. The newly 
Table I. Steady-state kinetic parameters for nucleotide incorporation opposite the normal G template by the full length and truncated mPolk proteins

\begin{tabular}{|c|c|c|c|c|c|c|}
\hline Polк & dNTP & $k_{\text {cat }}\left(\min ^{-1}\right)$ & $\boldsymbol{K}_{\mathbf{m}}(\mu \mathbf{M})$ & $k_{\text {cat }} / K_{\mathrm{m}}\left(\min ^{-1} \mu \mathbf{M}^{-1}\right)$ & $f_{\text {ins }}{ }^{a}$ & Relative efficiency ${ }^{b}$ \\
\hline \multirow{5}{*}{$1-852$} & A & $0.29 \pm 0.013$ & $91.5 \pm 20$ & $3.2 \times 10^{-3}$ & $7.9 \times 10^{-4}$ & \multirow{5}{*}{1} \\
\hline & $\mathrm{C}$ & $11.5 \pm 2.5$ & $2.86 \pm 1.2$ & 4 & 1 & \\
\hline & $\mathrm{T}$ & $0.43 \pm 0.018$ & $36.7 \pm 7.9$ & $1.2 \times 10^{-2}$ & $2.8 \times 10^{-3}$ & \\
\hline & G & $2.68 \pm 0.3$ & $311 \pm 21$ & $8.6 \times 10^{-3}$ & $2.1 \times 10^{-3}$ & \\
\hline & A & $1.11 \pm 0.04$ & $210 \pm 8.9$ & $5.3 \times 10^{-3}$ & $8.2 \times 10^{-4}$ & \\
\hline \multirow[t]{4}{*}{$1-516$} & $\mathrm{C}$ & $14.9 \pm 0.25$ & $2.31 \pm 0.34$ & 6.4 & 1 & \multirow[t]{4}{*}{1.6} \\
\hline & $\mathrm{T}$ & $0.71 \pm 0.11$ & $14.4 \pm 4.6$ & $4.9 \times 10^{-2}$ & $7.6 \times 10^{-3}$ & \\
\hline & G & $2.05 \pm 0.7$ & $170 \pm 12$ & $1.2 \times 10^{-2}$ & $1.9 \times 10^{-3}$ & \\
\hline & A & $0.73 \pm 0.16$ & $4354 \pm 465$ & $1.7 \times 10^{-4}$ & $3.8 \times 10^{-5}$ & \\
\hline \multirow{3}{*}{$22-516$} & $\mathrm{C}$ & $13.7 \pm 2.0$ & $3.10 \pm 1.5$ & 4.4 & 1 & \multirow[t]{3}{*}{1.1} \\
\hline & $\mathrm{T}$ & $0.35 \pm 0.06$ & $41.8 \pm 11$ & $8.4 \times 10^{-3}$ & $1.8 \times 10^{-3}$ & \\
\hline & $\mathrm{G}$ & $0.39 \pm 0.12$ & $2574 \pm 482$ & $1.5 \times 10^{-4}$ & $3.4 \times 10^{-5}$ & \\
\hline
\end{tabular}

${ }^{\mathrm{a}}$ Misinsertion frequency, calculated by dividing $k_{\text {cat }} / K_{\mathrm{m}}$ for each single dNTP incorporation by the $k_{\text {cat }} / K_{\mathrm{m}}$ for dCTP incorporation opposite undamaged G. ${ }^{\text {b }}$ Relative efficiency, calculated by dividing $k_{\text {cat }} / K_{\mathrm{m}}$ of truncated Pol $\kappa_{1-516}$ or Pol $\kappa_{22-516}$ for dCTP incorporation opposite normal $\mathrm{G}$ by that of $k_{\mathrm{cat}} / K_{\mathrm{m}}$ of the full length Pol $\kappa_{1-852}$.

prepared enzyme $(0-50 \mathrm{nM})$ was mixed with $0.2 \mu \mathrm{M}$ unlabelled and $20 \mathrm{nM}$ corresponding 6-FAM 5 -end labelled primer/template duplexes in the solution containing $25 \mathrm{mM}$ Tris- $\mathrm{HCl}(\mathrm{pH} 7.0)$, $0.1 \mathrm{mg} / \mathrm{ml}$ BSA, $1 \mathrm{mM}$ DTT, $5 \mathrm{mM} \mathrm{MgCl} 2,5 \mathrm{mM} \mathrm{NaCl}$ and $2.5 \%$ (wt $/ \mathrm{vol}$ ) glycerol. The reaction is preincubated at $37^{\circ} \mathrm{C}$ for $10 \mathrm{~min}$ and after initiation by the addition of dNTP mixture (30), the reaction was allowed to proceed at $37^{\circ} \mathrm{C}$ for indicated time slots. Products were terminated with $5 \mu \mathrm{l}$ stop solution containing $20 \mathrm{mM}$ EDTA, $95 \%$ formamide, $0.05 \%$ bromophenol blue and $0.05 \%$ xylene cyanol. After heating to $95^{\circ} \mathrm{C}$ for $3 \mathrm{~min}$, the extension products were separated on $15 \%$ denaturing polyacrylamide $(\mathrm{w} / \mathrm{v})$ gel, and quantified using Typhoon 9500 (GE Healthcare) and Image Quant version 5.2 (Amersham Biosciences).

\section{Steady-state kinetic analyses}

For the steady-state kinetic analysis, the 6-FAM labelled 24-nt primer 5'-GCATAGGGCGAATTCGGATGGTAG-3' was annealed with the unmodified or BPDE-modified 43-nt template at a primer/template ratio of $1: 1.2$, resulting the $24 / 43-n t$ duplex. The $3^{\prime}$ end of primer pairs with the template base $3^{\prime}$ preceding the adduct, and a single nucleotide insertion opposite the lesion was determined according to previously published methods (31-33). To examine extension from $\mathrm{G}$ or BPDE-dG paired with $\mathrm{C}$ template: primer termini, the 6-FAM labelled 25-nt primer 5'-GCATAGGG CGAATTCGGATGGTAGC- ${ }^{\prime}$ ' was annealed with the corresponding 43-nt template forming the 25/43-nt duplex, and the kinetic parameters were determined for single dNTP incorporation opposite the template base $\mathrm{C}$ immediately following the $\mathrm{G}$ or the BPDE-dG adduct. The polymerase concentrations $2-20 \mathrm{nM}$ enzyme(s), variable concentrations of a single nucleotide and reaction times 2,5 or $10 \mathrm{~min}$ were selected to ensure that the maximal product formation was $<20 \%(32,34)$. The reactions were terminated, subjected to denaturing PAGE and analysed using the Typhoon 9500. The parameters $k_{\mathrm{cat}}$ and $K_{\mathrm{m}}$ were extracted from the nonlinear regression fit of the Michaelis-Menten equation using Origin 8.0 software. All experiments were carried out in triplicate. Error bars indicate the standard deviation. The insertion efficiencies is indicated by $k_{\text {cat }} / K_{\mathrm{m}}$, and the misincorporation frequency $(f)$ is calculated from the expression $f_{\text {ins }}=\left(k_{\text {cat }} / K_{\mathrm{m}}\right)_{\mathrm{dNTP}} /\left(k_{\text {cat }} / K_{\mathrm{m}}\right)_{\mathrm{dCTP}}$.

\section{Results}

\section{Primer extension assays by the full-length and truncated $\mathrm{mPolk}$ proteins}

Full-length and both truncated Polк proteins $\left(\mathrm{Pol \kappa}_{1-516}\right.$ and Polk $22-516)$ were purified to near homogeneity using FPLC as previously reported (23) (Supplementary Fig. S1). Though at low enzyme concentration of $2 \mathrm{nM}$, Polк $\kappa_{22-516}$ exhibited less activity compared with Pol $\kappa_{1-852}$ and Pol $\kappa_{1-516}$, it readily extended the primer to the end of the normal DNA template at enzyme concentrations above $2 \mathrm{nM}$, as that of the other two proteins (Fig. 2B). A dominant 42-nt product in addition to a minor 43-nt was observed for Pol $\kappa_{1-852}$ and Pol $_{1-516}$, while Pol $\kappa_{22-516}$ exhibited extended 42-nt product only (Fig. 2C).

The presences of a BPDE-dG adduct retarded primer extension catalysed by different Polк. Full length and both truncated Polk extended the 22-nt primer by two nucleotides and paused immediately before the adduct at 24-nt (Fig. 2D). However, primer stalling of Pol $\kappa_{22-516}$ is highly pronounced compared with Pol $\kappa_{1-852}$ and Pol $\kappa_{1-516}$ proteins. When the polymerase concentration was increased from 2 to $50 \mathrm{nM}$, eventually all primers were extended to the end or near the end of the template strand by the full length and truncated Pol $\kappa_{1-516}$ protein (Fig. 2D), while Polא $\kappa_{22-516}$ displayed limited replication ability across the adduct. These data suggested that lack of the first 21 -aa leads to the inefficient lesion bypass by Pol $\kappa_{22}$ 516. However, Polא $22-516$ could extend the primer to the end when increasing the enzyme concentration and the extension product is in proportion to enzyme concentration. In addition, extension product length varied

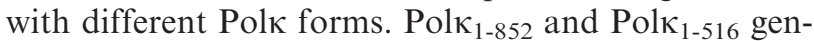
erated 42- and 43-nt extension products; and Polк $22-516$ predominantly produced 42- and a little 43-nt (Fig. 2E).

\section{Steady-state kinetic measurements of the full-length and Polk truncations}

Kinetic parameters $k_{\text {cat }}$ and $K_{\mathrm{m}}$ were measured first with the full length and truncated Polk proteins for single dNTP insertion opposite normal $G$ template (Table I), and the examples of typical single dCTP insertion assays were depicted in Figure 3A. Polא $\kappa_{1-516}$ behaved like Pol $\kappa_{1-852}$ on native DNA, and both are accurate in dCTP incorporation opposite $\mathrm{G}$, with misincorporation frequencies $\left[\left(k_{\mathrm{cat}} / K_{\mathrm{m}}\right)_{\text {incor }} /\left(k_{\mathrm{cat}} / K_{\mathrm{m}}\right)_{\mathrm{cor}}\right]$ in the order of $10^{-3}$ to $10^{-4}$ (Table I). When compared

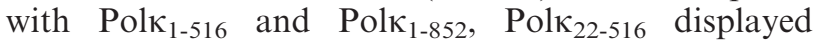
higher accuracy in incorporating single dCTP opposite undamaged $\mathrm{G}$, with misincorporation frequencies in 

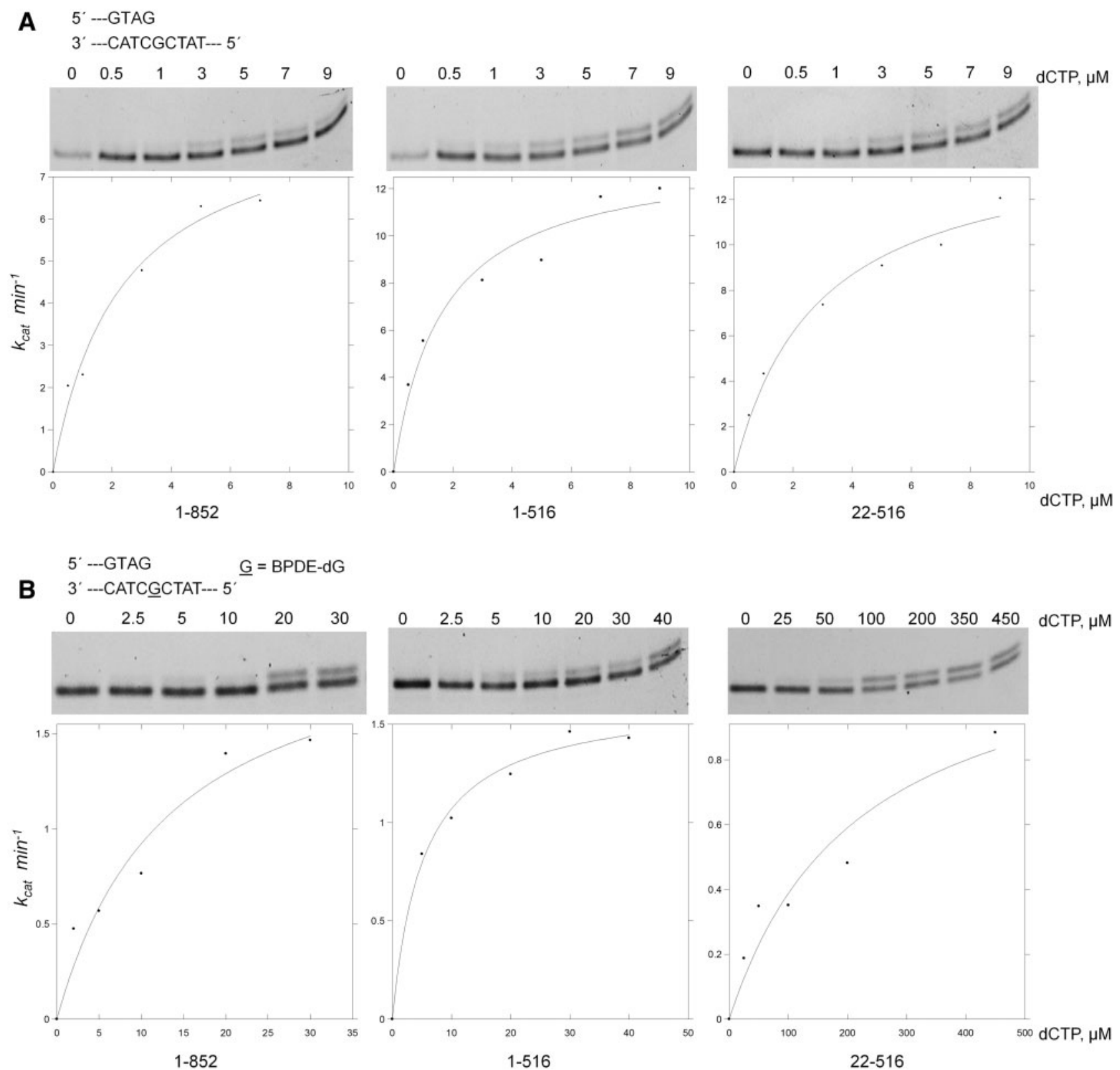

Fig. 3 Kinetics of single nucleotide insertion opposite (A) undamaged $\mathrm{G}$ or (B) BPDE-dG template by full length and truncated $\mathrm{mPolk}$. The gels (top panels) and plot (bottom panels) show data as a function of dCTP concentration. $0.2 \mu \mathrm{M}$ unlabelled and $20 \mathrm{nM}$ corresponding 6-FAM 5'-end labelled primer/template duplexes was incubated with $2 \mathrm{nM} \mathrm{Pol \kappa} \kappa_{1-852}$, Pol $\kappa_{1-516}$ or Pol $\kappa_{22-516}$ in the presence of dCTP for 2 min under the normal

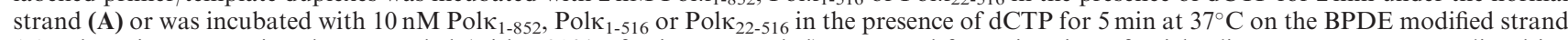
(B). The primer extension data recorded (with $<20 \%$ of primer extended) were used for estimation of Michaelis-Menten parameters listed in Tables I and II. Extended primers were separated by 15\% denaturing PAGE. Variants of mPolk mediated kinetics of single nucleotide incorporation on G or BPDE-dG by dATP, dTTP or dGTP were listed in Supplementary Figure S2.

the order of $10^{-3}$ to $10^{-5}$ (Table I). The greater reduction of misinsertion efficiencies $\left(k_{\mathrm{cat}} / K_{\mathrm{m}}\right)$ of dATP and dGTP of Pol $\kappa_{22-516}$ stems from increased $K_{\mathrm{m}}$ compared

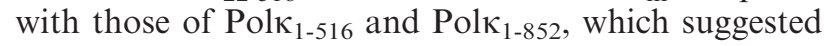
that the first 21 residues contribute additionally to the misinsertion efficiency while the catalytic efficiencies for correct nucleotide incorporation opposite unmodified template $\mathrm{G}$ are similar among different Polк forms (Table I).

$k_{\text {cat }}$ and $K_{\mathrm{m}}$ were also determined for the full-length and truncated Polא with respect to nucleotide insertion opposite the (+)-BPDE-dG adduct (Table II), and the representative single dCTP insertion experiments were shown in Figure 3B. When compared with Pol $\kappa_{1-852}$, Pol $\kappa_{22-516}$ catalytic core led to a decrease in the efficiency of dCTP insertion opposite a damaged $\mathrm{G}\left(k_{\mathrm{cat}} /\right.$ $K_{\mathrm{m}}$ ) by 28 -fold (Table II), which is consistent with the observation that Pol $\kappa_{22-516}$ is more severely blocked by BPDE-dG than Polא $\kappa_{1-852}$ in the running-start primer extension assay (Fig. 2D). On the other hand, Pol $\kappa_{1-516}$ exhibits 1.7-fold increase in dCTP insertion efficiency relative to that of the full length Polk (Table II).

The single dNTP insertion across the BPDE-dG

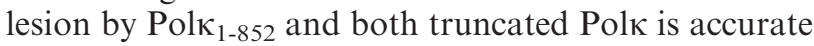
(Table II), the misinsertion frequencies of dATP and dTTP are decreased by two or three orders of 
Table II. Steady-state kinetic parameters for nucleotide incorporation opposite the (+)-trans-anti-BPDE-N ${ }^{2}$-dG adduct by the full length and truncated mPolk proteins ${ }^{\mathrm{a}, \mathrm{b}}$

\begin{tabular}{|c|c|c|c|c|c|c|}
\hline Polк & dNTP & $k_{\text {cat }}\left(\min ^{-1}\right)$ & $\boldsymbol{K}_{\mathbf{m}}(\mu \mathbf{M})$ & $k_{\text {cat }} / K_{\mathrm{m}}\left(\min ^{-1} \mu \mathbf{M}^{-1}\right)$ & $f_{\text {ins }}^{c}$ & Relative efficiency ${ }^{\mathrm{d}}$ \\
\hline \multirow{4}{*}{$1-852$} & A & $0.42 \pm 0.06$ & $326 \pm 75.3$ & $1.3 \times 10^{-3}$ & $8.5 \times 10^{-3}$ & \multirow{4}{*}{$3.8 \times 10^{-2}$} \\
\hline & $\mathrm{C}$ & $2.42 \pm 0.04$ & $16.0 \pm 3.54$ & $1.5 \times 10^{-1}$ & & \\
\hline & $\mathrm{T}$ & $0.35 \pm 0.01$ & $623 \pm 190$ & $5.6 \times 10^{-4}$ & $3.6 \times 10^{-3}$ & \\
\hline & A & $0.36 \pm 0.11$ & $103 \pm 6.05$ & $3.5 \times 10^{-3}$ & $1.3 \times 10^{-2}$ & \\
\hline \multirow[t]{2}{*}{$1-516$} & $\mathrm{C}$ & $1.64 \pm 0.24$ & $6.35 \pm 1.42$ & $2.6 \times 10^{-1}$ & 1 & \multirow[t]{2}{*}{$6.5 \times 10^{-2}$} \\
\hline & $\mathrm{T}$ & $0.35 \pm 0.05$ & $606 \pm 91.5$ & $5.8 \times 10^{-4}$ & $2.2 \times 10^{-3}$ & \\
\hline \multirow{3}{*}{$22-516$} & A & $0.11 \pm 0.03$ & $432 \pm 38.0$ & $2.5 \times 10^{-4}$ & $4.6 \times 10^{-2}$ & \multirow{3}{*}{$1.4 \times 10^{-3}$} \\
\hline & $\mathrm{C}$ & $1.14 \pm 0.35$ & $210 \pm 62.7$ & $5.4 \times 10^{-3}$ & 1 & \\
\hline & $\mathrm{T}$ & $0.18 \pm 0.04$ & $1906 \pm 285$ & $9.4 \times 10^{-5}$ & $1.7 \times 10^{-2}$ & \\
\hline
\end{tabular}

${ }^{a}$ A 24/43-nt duplex (sequence indicated in Fig. 1B) was used in the assay. ${ }^{b}$ No apparent incorporation of dGTP was observed even when high concentration of $2.5 \mathrm{mM}$ dNTP was used by the full length and the deletions. Thus, steady-state kinetic parameters were determined only for $\mathrm{dA}, \mathrm{dC}$ and dTTP incorporation. ${ }^{\mathrm{c}}$ Misinsertion frequency, calculated by dividing $k_{\text {cat }} / K_{\mathrm{m}}$ for each single dNTP incorporation by the $k_{\text {cat }} / K_{\mathrm{m}}$ for dCTP incorporation opposite BPDE-dG. ${ }^{\mathrm{d}}$ Relative efficiency, calculated by dividing $k_{\mathrm{cat}} / K_{\mathrm{m}}$ of Pol $\kappa_{1-852}$, truncated Pol $\kappa_{1-516}$ or Polk $\kappa_{22-516}$ for dCTP incorporation opposite BPDE-dG adduct by that of $k_{\mathrm{cat}} / K_{\mathrm{m}}$ of the full length Pol $\kappa_{1-852}$ for dCTP insertion opposite normal G in Table I.

Table III. Steady-state kinetic parameters for correct nucleotide dGTP incorporation opposite template base C immediately following G or BPDE-dG adduct by full-length, 1-516, and 22-516 mPolк $^{\mathrm{a}}$

\begin{tabular}{|c|c|c|c|c|}
\hline \multicolumn{5}{|c|}{ Normal G template, $25 / 43$-nt duplex } \\
\hline Polк & $k_{\text {cat }}\left(\min ^{-1}\right)$ & $\boldsymbol{K}_{\mathbf{m}}(\mu \mathbf{M})$ & $k_{\text {cat }} / K_{\mathrm{m}}\left(\min ^{-1} \mu \mathbf{M}^{-1}\right)$ & Relative to Polк ${ }_{1-852}{ }^{b}$ \\
\hline $1-852$ & $3.45 \pm 0.20$ & $0.684 \pm 0.150$ & 5.0 & 1 \\
\hline $1-516$ & $7.35 \pm 1.44$ & $0.948 \pm 0.737$ & 7.8 & 1.6 \\
\hline $22-516$ & $1.16 \pm 0.11$ & $0.912 \pm 0.284$ & 1.3 & $2.6 \times 10^{-1}$ \\
\hline \multicolumn{5}{|c|}{ BPDE-dG template, 25/43-nt duplex } \\
\hline Polк & $k_{\text {cat }}\left(\min ^{-1}\right)$ & $K_{\mathrm{m}}(\mu \mathbf{M})$ & $k_{\mathrm{cat}} / K_{\mathrm{m}}\left(\min ^{-1} \mu \mathrm{M}^{-1}\right)$ & Relative to Polк ${ }_{1-852}{ }^{\mathrm{c}}$ \\
\hline $1-852$ & $2.03 \pm 0.11$ & $10.6 \pm 2.36$ & $1.9 \times 10^{-1}$ & $3.8 \times 10^{-2}$ \\
\hline $1-516$ & $2.24 \pm 0.41$ & $3.62 \pm 0.57$ & $6.2 \times 10^{-1}$ & $1.2 \times 10^{-1}$ \\
\hline $22-516$ & $1.01 \pm 0.25$ & $39.2 \pm 0.50$ & $2.6 \times 10^{-2}$ & $5.2 \times 10^{-3}$ \\
\hline
\end{tabular}

${ }^{\mathrm{a}} \mathrm{A}$ 25/43-nt duplex (sequence indicated in Fig. 1B) was used in the assay. ${ }^{\mathrm{b}}$ Relative efficiency, calculated by dividing $k_{\mathrm{cat}} / K_{\mathrm{m}}$ of truncated Polk $_{1-516}$ or Polk $22-516$ for dGTP incorporation opposite template $\mathrm{C}$ immediately following normal G by that of $k_{\mathrm{cat}} / K_{\mathrm{m}}$ of the full length

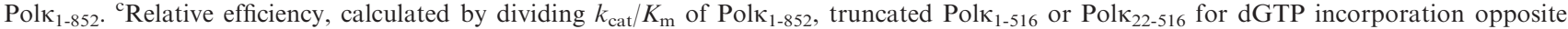
template C immediately following BPDE-dG adduct by that of $k_{\text {cat }} / K_{\mathrm{m}}$ of the full length Pol $\kappa_{1-852}$ for dGTP insertion opposite template C from the undamaged $\mathrm{G}: \mathrm{C}$ pair.

magnitude, respectively, compared with that of dCTP incorporation (Table II). An increased $K_{\mathrm{m}}$ compared with dCTP incorporation contributes to the decreased efficiencies of dATP or dTTP misinsertion by Pol $\kappa_{1-852}$

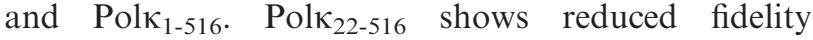

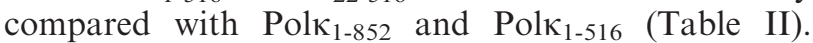

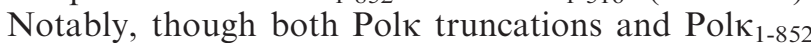
prefer to misincorporate dATP, Pol $\kappa_{22-516}$ exhibits increased frequency for dTTP misinsertion opposite BPDE-dG compared with that of the Pol $\kappa_{1-852}$ and Pol $\kappa_{1-516}$ by 5 - and 8 -fold, respectively (Table II).

\section{Extension by a single nucleotide beyond the undamaged and the BPDE-adducted $G$ with both the full length and truncated $\mathrm{mPolk}$}

In order to determine whether different primer extension patterns are present in the step immediately following dCTP insertion, we set out to measure the steady-state kinetics of base extension efficiency beyond the undamaged and BPDE damaged-dG. The kinetic parameters were determined for the next base extension from G: C or BPDE-dG: C pair of 25/43-nt duplex with different Polк forms (Table III). In the case of the next base extension from normal G: $\mathrm{C}$ template: primer pair termini, both the full length and truncated Polא $\kappa_{1-516}$ protein showed similar pattern to the correct nucleotide incorporation opposite undamaged G (Table I). Notably, the efficiency of dGTP insertion opposite template $\mathrm{C}$ immediately following the undamaged $\mathrm{G}$ by Polא $\kappa_{22-516}$ is reduced by 4 -fold compared with that of the Polk $1-852$ (Table III).

In addition, the kinetic parameters have also been measured for dGTP insertion opposite template $\mathrm{C}$ immediately following BPDE-dG (Table III). Pol $\kappa_{22-516}$ exhibits the lowest catalytic capability of dGTP incorporation compared with that of Pol $\kappa_{1-852}$ and Pol $\kappa_{1-516}$, as expected from both primer extension result (Fig. 2D) and steady-state kinetics of nucleotide insertion opposite the lesion (Table II). Together, these results suggest that deletion of the first 21 residues in Polk impairs both insertion and the subsequent extension step beyond the adduct. 


\section{Discussion}

The N-clasp, although seemingly dispensable for normal DNA synthesis and being structurally disordered $(16,17)$, plays critical roles in Polк's polymerase activity. In this study, we examined whether the extreme $\mathrm{N}$-terminal 21 residues in $\mathrm{mPol \kappa}$, closely adjacent to the $\mathrm{N}$-clasp, are required for its bypass of BPDE-dG lesions. Two truncated forms, Pol $\kappa_{1-516}$ and

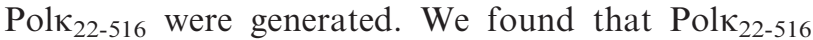
manifested an impaired TLS activity in bypass of the BPDE-DNA template and the deleted 21 residues contribute to the BPDE bypass efficiencies and subsequent extension proficiency.

Studies using the in vitro primer extension assay reveal that both Pol $\kappa_{1-516}$ and Pol $\kappa_{22-516}$ retain activities comparable to Pol $\kappa_{1-852}$ on normal DNA (Fig. 2B). Steady-state analyses indicate similar

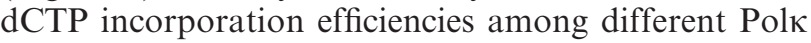
forms (Table I), which suggests that the first 21 residues are not necessary for normal DNA synthesis. When comparing the steady-state kinetic parameters for nucleotide extension from G:C pair template: primer termini using 25/43-nt duplex, we found Polk $22-516$ extends the primer less efficiently than Polk $1-516$ or Polk $\kappa_{1-852}$ (Table III), suggesting that the reduced frequency of Polא $\kappa_{22-516}$ for the next base extension beyond the undamaged $\mathrm{G}$ might explain its less activity under low concentration in running start primer extension assays as shown in Figure 2B. Anyway, the reduction could be complemented by increasing the enzyme concentration (Fig. 2B). Additionally, removal of the extreme N-terminal 21 residues of mPolk leads to a reduction in the misincorporation efficiencies of dATP or dGTP opposite undamaged $G$ (Table I), suggesting that the deletion influences the ability of Polk to discriminate purines during incorporation opposite the unmodified $G$.

Under the BPDE damaged template, Pol $\kappa_{22-516}$ manifests a strongly impaired TLS ability, leading to primer pausing in the vicinity of the adduct, while Polk $_{1-516}$ exhibits a higher TLS ability compared with that of the full length mPolк (Fig. 2D). The data indicate that the first 21 residues in mPolк are required for its optimal bypass of BPDE-dG lesion. The differences in polymerase stalling due to amino acid deletions are mirrored in the different catalytic efficiencies of dCTP incorporation opposite BPDE-dG (Table II), in next base extension immediately following the adduct (Table III) and in the overall TLS efficiencies (Supplementary Fig. S3) calculated by multiplying the relative efficiency for dCTP insertion opposite $G$ (or BPDE-G) (Tables I and II) and the relative efficiency for the next base extension (Table III) by different Polk as described previously $(35,36)$. When removal of the 1-21aa of Polk catalytic core, we see a 28 -fold decreases in correct dCTP incorporation efficiency opposite the BPDE-dG adduct compared with that of Pol $\kappa_{1-852}$, with the increase in $K_{\mathrm{m}}$ largely in charge of the reduction (Table II). Polk $\kappa_{22-516}$ also showed a 7 -fold decrease in $k_{\text {cat }} / K_{\mathrm{m}}$ for next base extension from the $G^{*}$ : C pair termini compared with that with the full length mPolк (Table III), suggesting that deletion influences Polк's activity for next base extension from both $\mathrm{G}$ : $\mathrm{C}$ and $\mathrm{G}^{*}$ : $\mathrm{C}$ pairs (Table III). The preference for misincorporating dATP is pronounced by both the full length protein and the truncations. The increase of misincorporation frequency of $\mathrm{dA}$ and $\mathrm{dT}$ under the BPDE-dG template is emphasized when removal of the first 1-21aa in the $\mathrm{N}$ terminus of mPolk (Table II). Lior-Hoffmann et al. (37) using molecular modelling and dynamic simulations, show that a hydrogen bond forms between the $\mathrm{BP}$ residue and Met135 in the full length Polк, hence a misincorporation of dTTP is less feasible, as demonstrated in previous work (20-22) and in Table II. When removal of the first 21 residues in $\mathrm{mPol \kappa}$, we suspect that the changes in Polא structural integrity and flexibility may permit the adducted template base $\mathrm{G}^{*}$ to position itself optimally for wobble pair geometry with dTTP, as we see 5- and 8-fold increase in misincorporation frequencies of dTTP opposite BPDE-dG compared with that of the Pol $\kappa_{1-852}$ and Pol $_{1-516}$, respectively (Table II).

Studies by Lone et al. (17) have shown that the first $18 \mathrm{~N}$-terminal amino acids of hPolк played important role in mismatch extension. hPolk minus the first 18 residues has severely diminished ability to extend mismatched primer template termini, while it retains primer extension proficiency from matched termini (17). On the undamaged DNA template, we found that Pol $\kappa_{22-516}$ retains similar proficiency for insertion correct dCTP and a lower efficiency for misincorporation opposite normal G (Table I), compared with that of full length Polk. These observations confirmed the conclusion by Lone et al. (17) that the first $18 \mathrm{~N}$-terminal residues contribute additionally to the misinsertion efficiency. In the case of BPDE-modified dG DNA, we found that compared with full-length protein, mPolк missing the first 21 residues exhibited greatly reduced ability in the translesion synthesis of the BPDE-dG lesion (Fig. $2 \mathrm{D}$, Table II), and the fidelity of the nucleotide incorporation opposite the lesion was reduced due to the deletions (Table II), suggesting that the amino acids of the Polк N-terminal region contribute to its TLS ability independent of DNA lesions.

Together, through biochemical comparison of these Polk truncated forms with the full length mPolк, we have found that deletion of the first 21 residues in mPolk severely impairs its efficiency and accuracy in bypass of the BPDE-DNA. The stalling of Pol $\kappa_{22-516}$ reflects not only on the nucleotide incorporation step but also on primer extension immediately after the lesion. Hence, an intact $\mathrm{N}$-terminus part is necessary for Polк to fulfill its optimal TLS functions opposite BPDE-dG lesions.

\section{Supplementary Data}

Supplementary Data are available at $J B$ Online.

\section{Acknowledgements}

We are grateful to Prof. Nicholas Geacintov for providing the 11-nt BPDE-modified strand, and Dr Wei Yang for helpful discussions about the project. 


\section{Funding}

This work was supported by the National Natural Science Foundation of China (Grant nos: 31300658, 31470784, 31170730, 31471331) and the Strategic Priority Research Program of the Chinese Academy of Sciences (XDB14030302).

\section{Conflict of Interest}

None declared.

\section{References}

1. Friedberg, E., Walker, G., Siede, W., Wood, R., Schultz, R., and Ellenberger, T. (2006) DNA Repair and Mutagenesis, 2nd edn. ASM Press, Washington, DC

2. Ohmori, H., Friedberg, E. C., Fuchs, R. P., Goodman, M. F., Hanaoka, F., Hinkle, D., Kunkel, T. A., Lawrence, C. W., Livneh, Z., Nohmi, T., Prakash, L., Prakash, S., Todo, T., Walker, G. C., Wang, Z., and Woodgate, R. (2001) The Y-family of DNA polymerases. Mol. Cell 8, 7-8

3. Yang, W. (2003) Damage repair DNA polymerases Y. Curr. Opin. Struct. Biol. 13, 23-30

4. Lange, S. S., Takata, K., and Wood, R. D. (2011) DNA polymerases and cancer. Nat. Rev. Cancer 11, 96-110

5. Yang, W. (2014) An Overview of Y-Family DNA Polymerases and a Case Study of Human DNA Polymerase eta. Biochemistry 53, 2793-2803.

6. Johnson, R. E., Prakash, S., and Prakash, L. (1999) Efficient bypass of a thymine-thymine dimer by yeast DNA polymerase, Poleta. Science 283, 1001-1004

7. Masutani, C., Kusumoto, R., Iwai, S., and Hanaoka, F. (2000) Mechanisms of accurate translesion synthesis by human DNA polymerase eta. EMBO J 19, 3100-3109

8. Biertumpfel, C., Zhao, Y., Kondo, Y., Ramon-Maiques, S., Gregory, M., Lee, J. Y., Masutani, C., Lehmann, A. R., Hanaoka, F., and Yang, W. (2010) Structure and mechanism of human DNA polymerase eta. Nature 465, 1044-1048

9. Masutani, C., Kusumoto, R., Yamada, A., Dohmae, N., Yokoi, M., Yuasa, M., Araki, M., Iwai, S., Takio, K., and Hanaoka, F. (1999) The XPV (xeroderma pigmentosum variant) gene encodes human DNA polymerase eta. Nature 399, 700-704

10. Johnson, R. E., Kondratick, C. M., Prakash, S., and Prakash, L. (1999) hRAD30 mutations in the variant form of xeroderma pigmentosum. Science 285, 263-265

11. Haracska, L., Johnson, R. E., Unk, I., Phillips, B. B., Hurwitz, J., Prakash, L., and Prakash, S. (2001) Targeting of human DNA polymerase iota to the replication machinery via interaction with PCNA. Proc. Natl. Acad. Sci. U. S. A. 98, 14256-14261

12. Washington, M. T., Minko, I. G., Johnson, R. E., Wolfle, W. T., Harris, T. M., Lloyd, R. S., Prakash, S., and Prakash, L. (2004) Efficient and error-free replication past a minor-groove DNA adduct by the sequential action of human DNA polymerases iota and kappa. Mol. Cell Biol. 24, 5687-5693

13. Choi, J. Y., Stover, J. S., Angel, K. C., Chowdhury, G., Rizzo, C. J., and Guengerich, F. P. (2006) Biochemical basis of genotoxicity of heterocyclic arylamine food mutagens: Human DNA polymerase eta selectively produces a two-base deletion in copying the N2-guanyl adduct of 2-amino-3-methylimidazo[4,5-f]quinoline but not the $\mathrm{C} 8$ adduct at the NarI G3 site. J. Biol. Chem. 281, 25297-25306

14. Kim, J., Song, I., Jo, A., Shin, J. H., Cho, H., Eoff, R. L., Guengerich, F. P., and Choi, J. Y. (2014) Biochemical analysis of six genetic variants of error-prone human DNA polymerase iota involved in translesion DNA synthesis. Chem. Res. Toxicol. 27, 1837-1852

15. Wojtaszek, J., Lee, C. J., D’Souza, S., Minesinger, B., Kim, H., D'Andrea, A. D., Walker, G. C., and Zhou, P. (2012) Structural basis of Rev1-mediated assembly of a quaternary vertebrate translesion polymerase complex consisting of Rev1, heterodimeric polymerase (Pol) zeta, and Pol kappa. J. Biol. Chem. 287, 33836-33846

16. Uljon, S. N., Johnson, R. E., Edwards, T. A., Prakash, S., Prakash, L., and Aggarwal, A. K. (2004) Crystal structure of the catalytic core of human DNA polymerase kappa. Structure 12, 1395-1404

17. Lone, S., Townson, S. A., Uljon, S. N., Johnson, R. E., Brahma, A., Nair, D. T., Prakash, S., Prakash, L., and Aggarwal, A. K. (2007) Human DNA polymerase kappa encircles DNA: implications for mismatch extension and lesion bypass. Mol. Cell 25, 601-614

18. Cheng, S. C., Hilton, B. D., Roman, J. M., and Dipple, A. (1989) DNA adducts from carcinogenic and noncarcinogenic enantiomers of benzo[a]pyrene dihydrodiol epoxide. Chem. Res. Toxicol. 2, 334-340

19. Geacintov, N. E., Cosman, M., Hingerty, B. E., Amin, S., Broyde, S., and Patel, D. J. (1997) NMR solution structures of stereoisometric covalent polycyclic aromatic carcinogen-DNA adduct: principles, patterns, and diversity. Chem. Res. Toxicol. 10, 111-146

20. Zhang, Y., Wu, X., Guo, D., Rechkoblit, O., and Wang, Z. (2002) Activities of human DNA polymerase kappa in response to the major benzo[a]pyrene DNA adduct: error-free lesion bypass and extension synthesis from opposite the lesion. DNA Repair (Amst) 1, 559-569

21. Rechkoblit, O., Zhang, Y., Guo, D., Wang, Z., Amin, S., Krzeminsky, J., Louneva, N., and Geacintov, N. E. (2002) trans-Lesion synthesis past bulky benzo[a]pyrene diol epoxide N2-dG and N6-dA lesions catalyzed by DNA bypass polymerases. J. Biol. Chem. 277, 30488-30494

22. Huang, X., Kolbanovskiy, A., Wu, X., Zhang, Y., Wang, Z., Zhuang, P., Amin, S., and Geacintov, N. E. (2003) Effects of base sequence context on translesion synthesis past a bulky (+)-trans-anti-B[a]P-N2-dG lesion catalyzed by the Y-family polymerase pol kappa. Biochemistry 42, 2456-2466

23. Liu, Y., Yang, Y., Tang, T. S., Zhang, H., Wang, Z., Friedberg, E., Yang, W., and Guo, C. (2014) Variants of mouse DNA polymerase kappa reveal a mechanism of efficient and accurate translesion synthesis past a benzo[a]pyrene dG adduct. Proc. Natl. Acad. Sci. U. S. A. 111, 1789-1794

24. Ogi, T., Shinkai, Y., Tanaka, K., and Ohmori, H. (2002) Polkappa protects mammalian cells against the lethal and mutagenic effects of benzo[a]pyrene. Proc. Natl. Acad. Sci. U. S. A. 99, 15548-15553

25. Avkin, S., Goldsmith, M., Velasco-Miguel, S., Geacintov, N., Friedberg, E. C., and Livneh, Z. (2004) Quantitative analysis of translesion DNA synthesis across a benzo[a]pyrene-guanine adduct in mammalian cells: the role of DNA polymerase kappa. J. Biol. Chem. 279, 53298-53305

26. Bi, X., Slater, D. M., Ohmori, H., and Vaziri, C. (2005) DNA polymerase kappa is specifically required for recovery from the benzo[a]pyrene-dihydrodiol epoxide (BPDE)-induced S-phase checkpoint. J. Biol. Chem. 280, 22343-22355 
27. Stancel, J. N. K., McDaniel, L. D., Velasco, S., Richardson, J., Guo, C. X., and Friedberg, E. C. (2009) Polk mutant mice have a spontaneous mutator phenotype. DNA Repair 8, 1355-1362

28. Mao, B., Xu, J., Li, B., Margulis, L. A., Smirnov, S., Ya, N. Q., Courtney, S. H., and Geacintov, N. E. (1995) Synthesis and characterization of covalent adducts derived from the binding of benzo[a]pyrene diol expoxide to a -GGG- sequence in a deoxyoligonucleotide. Carcinogenesis 16, 357-365

29. Guo, C., Fischhaber, P. L., Luk-Paszyc, M. J., Masuda, Y., Zhou, J., Kamiya, K., Kisker, C., and Friedberg, E. C. (2003) Mouse Rev1 protein interacts with multiple DNA polymerases involved in translesion DNA synthesis. EMBO J. 22, 6621-6630

30. Choi, J. Y., Angel, K. C., and Guengerich, F. P. (2006) Translesion synthesis across bulky N2-alkyl guanine DNA adducts by human DNA polymerase kappa. J. Biol. Chem. 281, 21062-21072

31. Johnson, R. E., Prakash, L., and Prakash, S. (2006) Yeast and human translesion DNA synthesis polymerases: expression, purification, and biochemical characterization. Methods Enzymol. 408, 390-407

32. Creighton, S., Bloom, L. B., and Goodman, M. F. (1995) Gel fidelity assay measuring nucleotide misinsertion, exonucleolytic proofreading, and lesion bypass efficiencies. Methods Enzymol. 262, 232-256
33. Benkovic, S. J., and Cameron, C. E. (1995) Kinetic analysis of nucleotide incorporation and misincorporation by Klenow fragment of Escherichia coli DNA polymerase I. Methods Enzymol. 262, 257-269

34. Goodman, M. F., Creighton, S., Bloom, L. B., and Petruska, J. (1993) Biochemical basis of DNA replication fidelity. Crit. Rev. Biochem. Mol. Biol. 28, 83-126

35. Song, I., Kim, E. J., Kim, I. H., Park, E. M., Lee, K. E., Shin, J. H., Guengerich, F. P., and Choi, J. Y. (2014) Biochemical characterization of eight genetic variants of human DNA polymerase kappa involved in error-free bypass across bulky N-guanyl DNA adducts. Chem. Res. Toxicol. 27, 919-930

36. Levine, R. L., Miller, H., Grollman, A., Ohashi, E., Ohmori, H., Masutani, C., Hanaoka, F., and Moriya, M. (2001) Translesion DNA synthesis catalyzed by human pol eta and pol kappa across 1,N6-ethenodeoxyadenosine. J. Biol. Chem. 276, 18717-18721

37. Lior-Hoffmann, L., Ding, S., Geacintov, N. E., Zhang, Y., and Broyde, S. (2014) Structural and dynamic characterization of polymerase kappa's minor groove lesion processing reveals how adduct topology impacts fidelity. Biochemistry 53, 5683-5691.

38. Ren, J., Wen, L., Gao, X., Jin, C., Xue, Y., and Yao, X. (2009) DOG 1.0: illustrator of protein domain structures. Cell Res. 19, 271-273 